Volume 19, No 3 International Journal of Radiation Research, July 2021

\title{
Investigating the out-of-field doses and estimating the risk of secondary thyroid cancer in high-grade gliomas radiation therapy with modulated intensity and 3D-conformal: a phantom study
}

\author{
S. Elmtalab and I. Abedi* \\ Department of Medical Physics, School of Medicine, Isfahan University of Medical Sciences, Isfahan, Iran
}

\section{- Original article}

\section{*Corresponding authors:}

Iraj Abedi, Ph.D.,

E-mail: i.abedi@med.mui.ac.ir

Revised: December 2020

Accepted: January 2021

Int. J. Radiat. Res., July 2021;

19(3): $569-574$

DOI: $10.29252 /$ ijrr.19.2.569

\begin{abstract}
Background: This study aimed to compare high-grade gliomas radiation therapy methods (high energy three-dimensional conformal radiation therapy [3D-CRT] vs. low energy intensity-modulated radiation therapy [IMRT]). Materials and Methods: Out-of-field photons resulting from 15- and 18-MV 3D-CRT and 6-MV IMRT techniques were measured with thermoluminescent dosimeters in a head and neck homogeneous phantom. Moreover, the dose to the left and right eye lenses, parotid glands, the thyroid gland, and the tongue was determined for each of the treatment techniques. Additionally, the risk of secondary thyroid cancer was estimated according to the biological effects of ionizing radiation BEIR VII model. Results: Errors in the treatment planning system (TPS) increased with increasing distance from the field edge and varied in different treatment techniques. The 6-MV IMRT technique increased the photon dose to all of the organs. Further, the excess relative risk for thyroid cancer was obtained higher in the 6-MV IMRT technique than in the 15- and 18-MV 3D-CRT techniques, as the photon dose to the thyroid gland was higher. Conclusion: Although 6-MV IMRT better improves local control and dose distribution than 3D-CRT, it increases the total dose equivalent in out-of-field organs independently of beam energy and exacerbates the risk of secondary thyroid cancer more significantly.
\end{abstract}

Keywords: Brain tumor, intensity-modulated radiotherapy, 3D-conformal radiotherapy, out-of-field dose, secondary cancer risk.

\section{INTRODUCTION}

Radiation therapy is an adjuvant treatment for malignant gliomas that increases median survival in patients (1). Any type of radiation therapy results in the out-of-field radiation of photons and treatment beams with energies above the threshold of reaction (photon, $\mathrm{n}$ ) result in neutrons (2-4). In the medical linear accelerator (LINAC), out-of-field photon radiation includes scattered photons of the collimator and the patient and the leakage of the LINAC head (5). Around the field edge, radiation scattering from both the patient and the collimator is the main source of out-of-field photon radiation. At a distance away from the field edge, the collimator leakage remains as radiation outside the field (6). Out-of-field doses in high-grade gliomas radiation therapy may induce side effects, mainly second cancers and eye abnormalities (7-9). Thyroid cancer is one of the most prevalent malignancies caused by neck and head radiation therapy $(10,11)$. Therefore, it is necessary to determine the photon dose to out-of-field critical organs. However, the treatment planning system (TPS) cannot estimate out-of-field photon doses and in-vivo dosimetry should be prescribed for measuring 
these doses (12).

Kourinou et al. (2013) (13) measured the dose to out-of-field organs in a pediatric phantom during 6-MV conventional brain radiation therapy. Majer etal. (2017) (14) conducted a study to compare the applications of 6-MV intensity-modulated radiation therapy (IMRT) and 6-MV three-dimensional conformal radiation therapy (3D-CRT) techniques in the same treatment area. Ahmadi Khui et al. (2017) (15) calculated the thyroid gland dose and the risk of secondary thyroid cancer in whole-brain 3D-CRT with 6-MV X-rays.

Although several studies have been conducted to measure or calculate out-of-field photon doses and estimate the risk of secondary thyroid cancer during the treatment of brain tumors, less attention has been paid to different energies, especially high energies, of 3D-CRT and IMRT treatment techniques.

This study aimed to compare high energy 3D-CRT and low energy IMRT as high-grade gliomas radiation therapy methods. Out-of-field photon doses were measured in a standard adult phantom and the risk of secondary thyroid cancer was estimated.

\section{MATERIALS AND METHODS}

\section{Phantom}

A homogeneous tissue-equivalent phantom was used to measure photon doses. The phantom was designed based on the CT (Siemens 64-Slice) image data of a standard adult patient collected at the Milad Hospital, Isfahan, Iran, using 3D-Doctor software (FDA $510 \mathrm{~K}$ Cleared). All the phantom pieces were produced from a Perspex sheet (density: 1.18 $\mathrm{gr} / \mathrm{cm}^{3}$, Zeff: 6.48 , and thickness: $2.7 \mathrm{~cm}$ ) (16) using an automatic laser cutter (Crystal Sign, EZ-T1490Z).

\section{Contouring}

The simulated gross tumor volume (GTV) was determined in the right temporal lobe of the brain $\left(\mathrm{V}_{\mathrm{GTV}}\right.$ : $\left.5087 \mathrm{~cm}^{3}\right)$. The planning target volume (PTV) was created by adding a $1-\mathrm{cm}$ expansion to GTV to account for set up 570 uncertainties. Target volumes, OAR structures such as brainstem, optic chiasm, hippocampus, optic nerves, orbits, and out-of-field organs (section 2.4.1) were outlined by a radiation oncologist in TPS (PROWESS, version 5.5).

\section{Treatment planning}

For 3D-CRT plans, a three-field arrangement including a vertex field and opposed laterals was utilized for 15- and 18-MV X-rays to prepare a dose of 54 Gy in 1.8Gy fractions to the tumor in case $95 \%$ of PTV was received as a minimum of $95 \%$ of the prescribed dose and critical organs received an acceptable dose (table 1). The 3D-CRT plans were retrieved on the same conditions to generate an IMRT plan consisting of a seven-field arrangement with beam angles of $0,60,90,120,240,270$, and $300^{\circ}$ for $6-\mathrm{MV}$ $\mathrm{X}$-rays using an inverse planning system (PROWESS).

Table 1. The dose limits for OAR organs ${ }^{(17,18)}$.

\begin{tabular}{|c|c|}
\hline Organ & Dose limitation [cGy] \\
\hline Hippocampus & $D_{\max }<600$ \\
\hline Optic nerves & $D_{\max }<5500$ \\
\hline Chiasm & $D_{\max }<5600$ \\
\hline Brainstem & $D_{\max }<6000$ \\
\hline Retina & $D_{\max }<5000$ \\
\hline
\end{tabular}

The 6-MV IMRT and 15-MV 3D-CRT plans were performed using Siemens-Artiste 5918 LINAC and the Siemens-Oncor 5099 LINAC was used for 18-MV 3D-CRT. Figures 1 and 2 show dose distribution and MLC apertures for the plans, respectively.

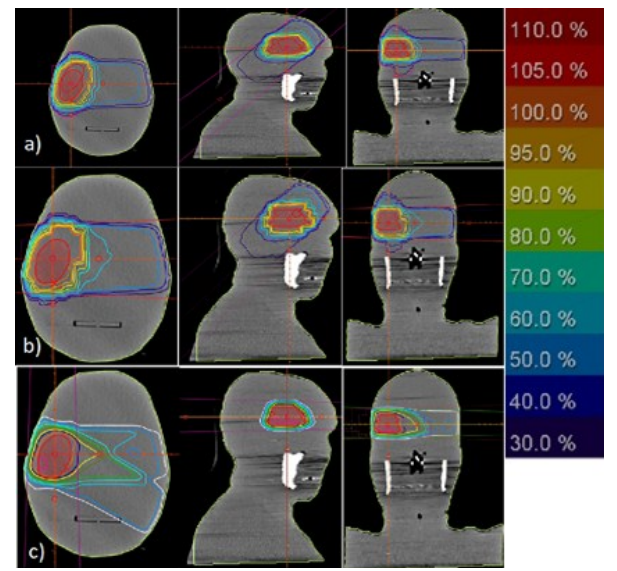

Figure 1. Dose distributions for a) $15 \mathrm{MV}$ 3D-CRT, b) $18 \mathrm{MV}$ 3D-CRT, and c) $6 \mathrm{MV}$ IMRT treatment plans in phantom.

Int. J. Radiat. Res., Vol. 19 No. 3, July 2021 


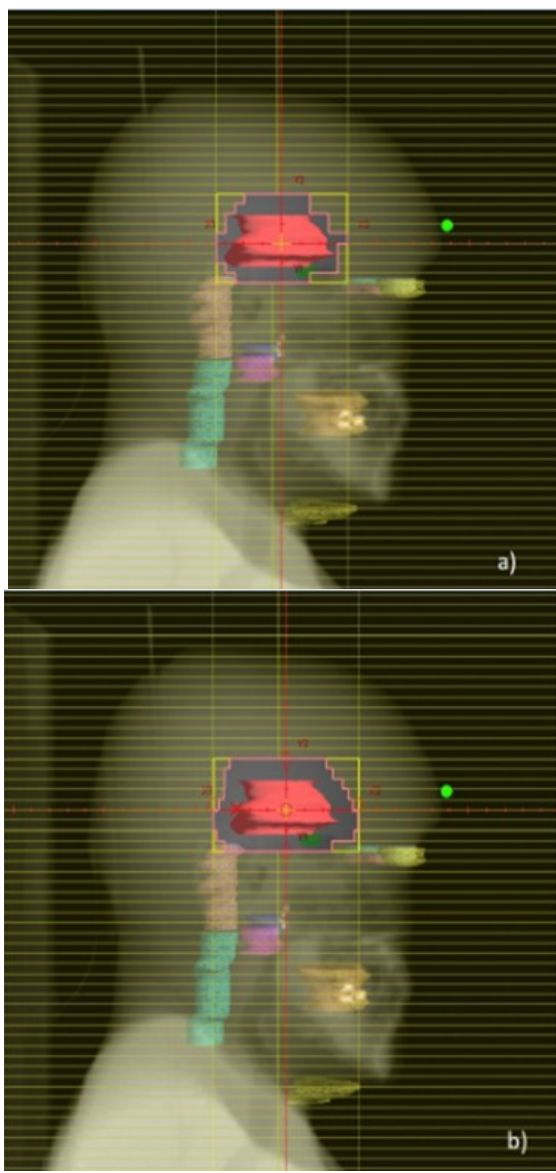

Figure 2. The MLC apertures projected on right sagittal a) for the Siemens-Oncor 5099 and b) the Siemens-Artist 5918.

\section{Measurement \\ TLD calibration}

Lithium fluoride thermo-luminescent dosimeter (TLD-700, Harshaw Chemical Company, USA) chips were utilized to measure photon doses in the phantom. TLD-700 is independent of neutrons, and measures photon dose with an error rate of $1 \%$ (2).

The TLD chips were annealed at $400^{\circ} \mathrm{C}$ for $1 \mathrm{~h}$ and $100^{\circ} \mathrm{C}$ for $2 \mathrm{~h}$ before every irradiation. A $1.6-\mathrm{cm}$ slab of water phantom (PMMA) was placed on the TLD chips to make an electronic e quilibrium for batch and individual calibration. Then, the chips were irradiated on appropriate conditions (field size: $10 \times 10 \mathrm{~cm}^{2}$ and SSD: 100 $\mathrm{cm}$ ). The individual calibration factor (ICF) was selected to examine the intrinsic differences of the chips and the batch calibration factor (BCF) was determined to obtain readings in terms of dose units (19-22).

\section{Measurements}

The out-of-field organs included the left and right eye lenses, parotid glands, the tongue, and the thyroid gland. The three-dimensional coordinates of the center of the out-of-field organs were determined in TPS (figure 3 ). The TLD chips were located at these coordinates on the phantom and three TLD readings were performed.

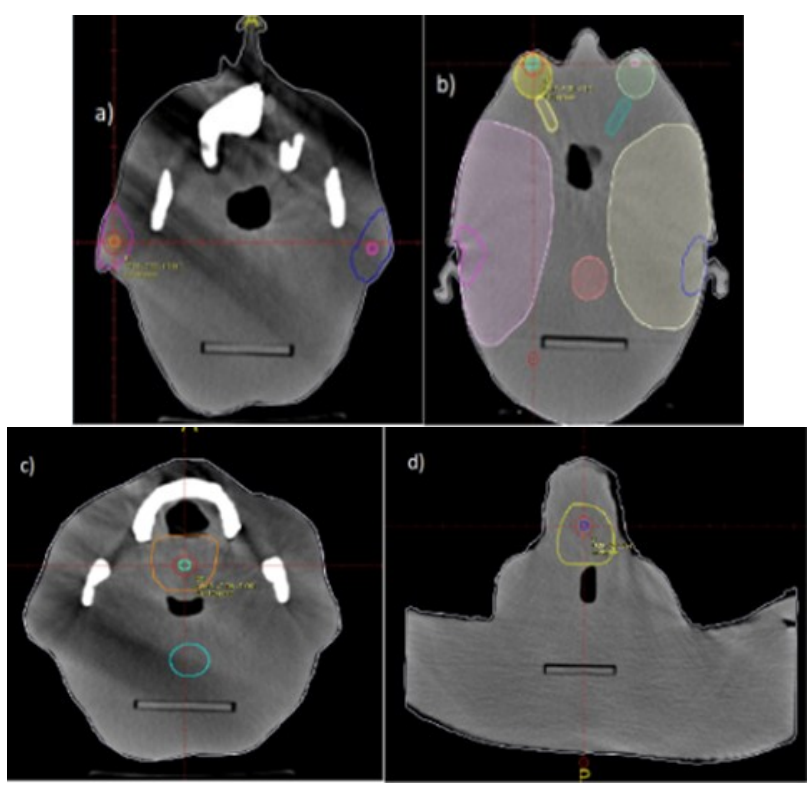

Figure 2. The MLC apertures projected on right sagittal a) for the Siemens-Oncor 5099 and b) the Siemens-Artist 5918.

The absorbed dose in each point was calculated using equation 1 :

Dose $=\left[\mathrm{cc}_{\text {dose }}-\mathrm{cc}_{\mathrm{bdg}}\right] \times \mathrm{BCF} \times \mathrm{ICF}$

where $\mathrm{CC}_{\text {dose, }}, \mathrm{Cc}_{\mathrm{bdg}}, \mathrm{BCF}$, and ICF are the TLD reading, the background TLD reading, the batch calibration factor, and the individual calibration factor, respectively (19-22).

\section{Estimation of thyroid cancer risk}

In the current study, thyroid cancer risk was approximated regarding the measured dose using the biological effects of ionizing radiation (BEIR) VII model.

The BIER VII Committee estimated excess absolute risk (EAR) and excess relative risk (ERR) to report the risk of radiogenic cancer for particular organs. ERR represents the cancer 
rate (cancers per 10,000 people) related to the background radiation level, while EAR is merely the difference in radiation-attributed rates. ERR and EAR are functions of age-at-exposure, attained age, and sex (23). For thyroid cancer, the BEIR VII model utilized merely ERR to quantify the risk of the cancer, which is calculated using equation 2:

$\left.\operatorname{ERR}(\mathrm{D}, \mathrm{S}, \mathrm{e})=\beta_{\mathrm{S}} \operatorname{Dexp}\left[\gamma^{\left(e^{-30} / 10\right.}\right)\right](a / 60)^{n}$

Where; e: age at exposure, D: absorbed dose ,a: attained age (ERR is independent of attained age)

Constant coefficients $\beta \mathrm{s}, \gamma$, and $\mathrm{n}$ were obtained from table 3-3 in reference (23).

\section{RESULTS}

Table 2 shows doses determined using TLD-700 ( $\left.\mathrm{D}_{\text {meas }}\right)$, doses calculated using prowess TPS ( $\left.\mathrm{D}_{\text {cal }}\right)$, and the differences (\%) between them for 6-MV IMRT and 15- and 18-MV 3D-CRT. The findings indicated that TPS underestimated out-of-field photon doses. Error rates in TPS increased for the distances of 0.3 to $11.9 \mathrm{~cm}$ from the field edge by incrementing the distance from the field edge and varied in the different treatment techniques .Additionally, the out-offield photon doses decreased sharply with an increase in the distance from the field edge. Moreover, in 6-MV IMRT, the organs received higher doses due to off-field photons.

Table 2. Dmeas (TLD) and Dcal (TPS) in the center of out-of-field organs and TPS error for different treatment techniques.

\begin{tabular}{|c|c|c|c|c|c|c|c|c|c|c|}
\hline \multirow{3}{*}{ organs } & \multirow{3}{*}{$\begin{array}{l}\text { Distance (from } \\
\text { the edge of the } \\
\text { field) }[\mathrm{cm}]\end{array}$} & \multirow{2}{*}{\multicolumn{3}{|c|}{$\begin{array}{l}\text { Siemens-Oncor } \\
18 \text { MV 3D-CRT }\end{array}$}} & \multicolumn{6}{|c|}{ Siemens-Artiste } \\
\hline & & & & & \multicolumn{3}{|c|}{15 MV 3D-CRT } & \multicolumn{3}{|c|}{$6 \mathrm{MV}$ IMRT } \\
\hline & & $D_{\text {meas }}[\mathrm{CGy}]$ & $\mathrm{D}_{\text {cal }}[\mathrm{cGy}]$ & $\%$ & $D_{\text {meas }}[c G y]$ & $\mathrm{D}_{\text {cal }}[\mathrm{CGy}]$ & $\%$ & $D_{\text {meas }}[c G y]$ & $D_{\text {cal }}[\mathrm{cGy}]$ & $\%$ \\
\hline $\begin{array}{c}\text { Right parotid } \\
\text { gland }\end{array}$ & 0.3 & $140.0 \pm 1.6^{*}$ & 131.6 & $-6 \%$ & $136.0 \pm 1.7$ & 131.9 & $-3 \%$ & $144 \pm 1.2$ & 129.6 & $-10 \%$ \\
\hline Right eye lens & 3 & $9 \pm 0.9$ & 7.8 & $-13 \%$ & $49.3 \pm 0.9$ & 44.0 & $-10.7 \%$ & $84.2 \pm 1.1$ & 61.5 & $-26.9 \%$ \\
\hline Left eye lens & 6 & $43.5 \pm 1.0$ & 5.7 & $-18 \%$ & $38.5 \pm 0.9$ & 32.2 & $-16.4 \%$ & $57.2 \pm 1.2$ & 35.2 & $-38.5 \%$ \\
\hline $\begin{array}{l}\text { Left parotid } \\
\text { gland }\end{array}$ & 7.2 & $37.0 \pm 1.0$ & 31.1 & $-15.9 \%$ & $33.0 \pm 0.9$ & 28.2 & $-14.6 \%$ & $45.8 \pm 1.1$ & 29.0 & $-36.7 \%$ \\
\hline Tongue & & $18.2 \pm 0.6$ & 14.7 & $-19.3 \%$ & $15.1 \pm 0.5$ & 12.4 & $-18 \%$ & $24.5 \pm 0.7$ & 13.7 & $-44 \%$ \\
\hline Thyroid gland & 11.9 & $6.8 \pm 0.3$ & 5.1 & $-25 \%$ & $3.7 \pm 0.5$ & 2.8 & $-24.3 \%$ & $9.5 \pm 0.4$ & 3.9 & $-58.9 \%$ \\
\hline
\end{tabular}

Table 3 shows ERR for thyroid cancer in 6-MV IMRT and 15- and 18-MV 3D-CRT of both sexes at the exposure age of 20-60 years. The risk of secondary thyroid cancer was higher in 6-MV IMRT due to the out-of-field photon dose.

Table 3. The ERR of thyroid cancer for adult in different treatment techniques.

\begin{tabular}{|c|c|c|c|c|c|c|}
\hline Age & \multicolumn{2}{|c|}{ 18 MV 3D-CRT } & \multicolumn{2}{c|}{ 15 MV 3D-CRT } & \multicolumn{2}{c|}{ 6 MV IMRT } \\
\hline & Male & Female & Male & Female & Male & Female \\
\hline $\mathbf{2 0}$ & $0.083 \pm 0.003^{*}$ & $0.164 \pm 0.007$ & $0.045 \pm 0.006$ & $0.089 \pm 0.012$ & $0.115 \pm 0.005$ & $0.229 \pm 0.009$ \\
\hline $\mathbf{3 0}$ & $0.036 \pm 0.001$ & $0.071 \pm 0.003$ & $0.020 \pm 0.002$ & $0.039 \pm 0.005$ & $0.050 \pm 0.002$ & $0.100 \pm 0.004$ \\
\hline $\mathbf{4 0}$ & $0.016 \pm 0.000$ & $0.031 \pm 0.001$ & $0.009 \pm 0.001$ & $0.017 \pm 0.002$ & $0.022 \pm 0.009$ & $0.043 \pm 0.001$ \\
\hline $\mathbf{5 0}$ & $0.007 \pm 0.000$ & $0.014 \pm 0.000$ & $0.004 \pm 0.013$ & $0.007 \pm 0.001$ & $0.001 \pm 0.000$ & $0.019 \pm 0.001$ \\
\hline $\mathbf{6 0}$ & $0.003 \pm 0.000$ & $0.006 \pm 0.000$ & $0.002 \pm 0.000$ & $0.003 \pm 0.000$ & $0.008 \pm 0.000$ & $0.004 \pm 0.000$ \\
\hline
\end{tabular}

\section{DISCUSSION}

In this study, 15- and 18-MV 3D-CRT and 6-MV IMRT treatment techniques for out-of-field photon doses and the risk of secondary thyroid cancer were examined and compared.
In previous studies, Foo et al. (1993) (24) investigated out-of-field doses during brain treatment with conventional treatment techniques. In total, 50 cGy was prepared to the isocenter using Cobalt- 60 gamma rays and 6 and 18 MV X-rays. The left and right eye lenses Int. J. Radiat. Res., Vol. 19 No. 3, July 2021 
received equal 40-200 cGy doses of radiation. In this study, the dose to the eye lenses ranged from 38.5 to 59.9 cGy for 3D-CRT. Principally, due to the proximity to the field edge in the vertex field, the dose to the right eye lens was higher than that to the left eye lens. The thyroid gland doses (350-600 cGy) in Foo et al.'s study were higher than those reported in our study. This poor agreement could be due to differences in the vertex fields. Ahmadi Khui et al. (2017) (15) measured the thyroid gland absorption dose in 50 patients with the mean age less than 30 years undergoing whole-brain radiation using 6-MV 3D-CRT. They reported the minimum and maximum doses as 0.941 cGy and 6.028 cGy, respectively. This is in good agreement with the present findings and confirms the results of Ruben et al.'s study (2014) (25), claiming that in a treatment technique (3D-CRT or IMRT), the out-of-field dose is almost independent of the therapeutic energy. Kourinou et al. (2013) (13) determined the scatter dose to out-of-field organs from brain radiotherapy in 5- and 10-year-old phantoms. The plan consisting of two lateral and opposed treatment fields was irradiated with 6-MV beam. The thyroid gland dose was obtained 12.48 and 11.26 cGy for the 5- and 10-year-old phantoms, respectively. Therefore, the thyroid gland dose was higher for the younger patients. According to Majer et al.'s (2017) study (14), out-of-field organ doses are on average 1.6 times higher for a 5-year-old phantom than for a 10 -year-old phantom. Since an adult phantom was used in the present study, the reduction in the thyroid dose compared to Kourinou's report is justified. Majer et al. measured out-of-field organ doses for 3D-CRT and IMRT of brain tumors. The thyroid gland dose was obtained less than $1 \%$ of the treatment dose, which confirms the findings of the present study.

Shore etal. (1992) (26) reported that low thyroid gland doses of 10 cGy could result in secondary malignancies. Therefore, the thyroid gland doses obtained in this study are not negligible. Acun et al. (2007) (27) in their dosimetric study on a phantom reported the ERR of thyroid cancer as 0.1 in adults during total cranial irradiation, which is in agreement with the current findings.

High energy treatments provide excellent skin-sparing, accurate penetration, uniform spatial dose distribution, sharp field edges, and small penumbra (28). The use of high energy beams for 3D-CRT to provide a uniform dose distribution can produce neutron contamination (29). However, low energy IMRT treatment for high-grade gliomas can improve dose conformity and dose distribution without increasing integral dose (30) and unwanted neutron dose in off-field organs. On the other hand, it has been found that, in general, IMRT has more out-of-field photon doses than 3D-CRT because of greater collimator scatter, leakage, and the number of radiation fields in IMRT (25). This study's results showed that this principle was independent of beam energy and 6-MV IMRT caused higher out-offield photon doses than 15- and 18-MV 3D-CRT in all the considered organs during brain tumor radiation therapy. However, it is best to consider the neutron contamination dose in the organs. In Elmtalab et al.'s study (2020) (31), the neutron dose equivalent to thyroid gland was reported $12.3 \mathrm{mSv}$ for the prescribed dose of $54 \mathrm{~Gy}$ in brain radiation therapy with $15-\mathrm{MV}$ X-rays, which was insignificant compared to the received photon dose in this study.

\section{CONCLUSION}

Although low energy IMRT can improve local control and dose distribution without high integral dose and neutron contamination, it produces higher off-field photon doses than high energy 3D-CRT. On the other hand, the unwanted neutron-induced dose is insignificant in high energy 3D-CRT because the neutron to scattered photon ratio is minimal. Therefore, it can be concluded that IMRT produces a higher total dose equivalent than 3D-CRT in out-of-field organs, independently of beam energy in brain radiation therapy, and consequently increases the risk of secondary cancers such as thyroid cancer.

Conflicts of interest: Declared none. 


\section{REFERENCES}

1. Andersen AP (1978) Postoperative irradiation of glioblastomas: Results in a randomized series. Acta Radiol Oncol Radiat Phys Biol, 17(6): 475-84.

2. Kry SF, Salehpour M, Followill DS, et al. (2005) Out-of-field photon and neutron dose equivalents from step-andshoot intensity-modulated radiation therapy. Int J Radiat Oncol Biol Phys, 62(4): 1204-16.

3. American Association of Physicists in Medicine (1986) Neutron measurements around high energy $\mathrm{X}$-ray radiotherapy machines. American Institute of Physics for American Association of Physicists in Medicine, United States.

4. Howell RM, Hertel NE, Wang Z, et al. (2006) Calculation of effective dose from measurements of secondary neutron spectra and scattered photon dose from dynamic MLC IMRT for, and beam energies. Med Phys, 33(2): 360-8.

5. Cozzi L, Buffa FM, Fogliata A (2001) Dosimetric features of linac head and phantom scattered radiation outside the clinical photon beam: experimental measurements and comparison with treatment planning system calculations. Radiother Oncol, 58(2): 193-200.

6. Kase KR, Svensson GK, Wolbarst AB, et al. (1983) Measurements of dose from secondary radiation outside a treatment field. Int J Radiat Oncol Biol Phys, 9(8): 1177-83.

7. Ron E (2003) Cancer risks from medical radiation. Health Phys, 85(1): 47-59.

8. Van der Laan B, Baris G, Gregor RT, et al. (1995) Radiationinduced tumours of the head and neck. J Laryngol Otol, 109(4): 346-9.

9. Hempel $M$ and Hinkelbein W (1993) Eye sequelae following external irradiation. Recent Results Cancer Res, 130: 231-236.

10. Rubino C, De Vathaire F, Dottorini M, et al. (2003) Second primary malignancies in thyroid cancer patients. $\mathrm{Br}$ J Cancer, 89(9): 1638-44.

11. Rose J, Wertheim BC, Guerrero MA (2012) Radiation treatment of patients with primary pediatric malignancies: risk of developing thyroid cancer as a secondary malignancy. Am J Surg, 204(6): 881-7.

12. Howell RM, Scarboro SB, Kry SF, et al. (2010) Accuracy of out-of-field dose calculations by a commercial treatment planning system. Phys Med Biol, 55(23): 6999.

13. Kourinou KM, Mazonakis M, Lyraraki E, et al. (2013) Scattered dose to radiosensitive organs and associated risk for cancer development from head and neck radiotherapy in pediatric patients. Phys Med, 29(6): 650-5.

14. Majer M, Stolarczyk L, De Saint-Hubert M, et al. (2017) Out-of-field dose measurements for 3D conformal and intensity modulated radiotherapy of a paediatric brain tumour. Radiat Prot Dosim, 176(3): 331-40.

15. Ahmadi M, Tavakoli MB, Amouheidari A, et al. (2017) Investigation of the absorbed dose and estimation of the risk of secondary thyroid cancer in whole-brain radiotherapy. J Isfahan Med Sch, 34(413): 1590-1594.

16. Khan FM and Gibbons JP (2014) Khan's the physics of radiation therapy. LWW, United States.
17. Cox JD, Stetz J, Pajak TF (1995) Toxicity criteria of the radiation therapy oncology group (RTOG) and the European organization for research and treatment of cancer (EORTC). Int J Radiat Oncol Biol Phys, 31(5): 1341-6.

18. Scoccianti S, Detti B, Gadda D, et al. (2015) Organs at risk in the brain and their dose-constraints in adults and in children: a radiation oncologist's guide for delineation in everyday practice. Radiother Oncol, 114(2): 230-8.

19. Banaee N and Nedaie HA (2013) Evaluating the effect of energy on calibration of thermo-luminescent dosimeters 7 -LiF: Mg, Cu, P (GR-207A). Int J Radiat Res, 11(1): 51.

20. Moafi M, Geraily G, Shirazi AR (2019) Comparison of thermoluminescent dosimeter calibration irradiated in gamma knife and 60Co instruments. J Cancer Res Ther, 15(8): 123.

21. Nazem MNM, Shahbazi-Gahrouei $D$, saleh Jafarpishe $M$, et al. (2018) Evaluation of cumulative radiation dose in neonates in neonatal surgery ward. Iran J Pediatr, 4(2): 71-9.

22. Meinhold CB, Abrahamson S, Adelstein SJ, et al. (1993) Limitation of exposure to ionizing radiation. NCRP REP, (116): 1-86.

23. National Research Council (2006) Health risks from exposure to low levels of ionizing radiation: BEIR VII phase 2 . NAP, United States.

24. Foo ML, McCullough EC, Foote RL, et al. (1993) Doses to radiation sensitive organs and structures located outside the radiotherapeutic target volume for four treatment situations. Int J Radiat Oncol Biol Phys, 27(2): 403-17.

25. Ruben JD, Smith R, Lancaster CM, et al. (2014) Constituent components of out-of-field scatter dose for 18-MV intensity modulated radiation therapy versus 3-dimensional conformal radiation therapy: a comparison with 6-MV and implications for carcinogenesis. Int I Radiat Oncol Biol Phys, 90(3): 645-53.

26. Shore RE (1992) Issues and epidemiological evidence regarding radiation-induced thyroid cancer. Radiat Res, 131 (1): 98-111.

27. Acun H, Kemikler G, Karadeniz A (2007) Dosimetric analysis of thyroid doses from total cranial irradiation. Radiat Prot Dosimetry, 123(4): 498-504.

28. Das IJ and Kase KR (1992) Higher energy: is it necessary, is it worth the cost for radiation oncology? Med Phys, 19(4): 917-25.

29. Alfuraih A, Chin MP, Spyrou NM (2008) Measurements of the photonuclear neutron yield of $15 \mathrm{MV}$ medical linear accelerator. J Radioanal Nucl Chemis, 278(3): 681-4.

30. Hermanto U, Frija EK, Lii MJ, Chang EL, et al. (2007) Intensity modulated radiotherapy (IMRT) and conventional three-dimensional conformal radiotherapy for high-grade gliomas: Does IMRT increase the integral dose to normal brain? Int J Radiat Oncol Biol Phys, 67(4): 1135-44.

31. Elmtalab S, Abedi I, Choopan-Dastjerdi M, et al. (2020) Evaluation of risk of secondary thyroid cancer caused by neutron dose equivalent from brain tumor 3D-conformal radiation therapy (3D-CRT). J Isfahan Med Sch, 38(586): 569-574.

Int. J. Radiat. Res., Vol. 19 No. 3, July 2021 